\title{
Sulcus-Deepening Trochleoplasty as an Isolated or Combined Treatment Strategy for Patellar Instability and Trochlear Dysplasia: A Systematic Review
}

\author{
Michael Robert Davies, M.D., Sachin Allahabadi, M.D., Tarek Elliott Diab, \\ Ryan David Freshman, M.D., Nirav Kiritkumar Pandya, M.D., Brian Thomas Feeley, M.D., \\ and Drew Anderson Lansdown, M.D.
}

\begin{abstract}
Purpose: To highlight the indications and outcomes for sulcus-deepening trochleoplasty, when used as an isolated procedure as well as in combination with other stabilization techniques for patellar instability. Methods: We performed a systematic review focused on outcomes and complications following trochleoplasty performed either as an isolated procedure or in combination with other procedures to address patellar instability. Inclusion criteria included studies in English that reported on outcomes following primary open trochleoplasty, including Kujala scores and recurrent instability or dislocation events. Results: Twelve papers including 702 patients who underwent sulcus-deepening trochleoplasty were included. A total of 504 patients underwent isolated sulcus-deepening trochleoplasty, whereas 198 patients underwent trochleoplasty in combination with 1 or more additional stabilization procedures. In total, $67 \%$ of patients were female compared with 33\% male. The procedure was done was a primary surgical intervention $74 \%$ of the time. Postoperative Kujala scores for isolated trochleoplasty ranged from 80 to 92, whereas those for combined stabilization procedures ranged from 76 to 95 . The dislocation rate among the studies ranged from 0 to $8 \%$. There was a persistent $\mathrm{J}$-sign in 0 to $12 \%$ of treated knees among all studies, and a persistent apprehension test in 0 to $29 \%$ of treated knees. Return to play ranged from $65 \%$ to $83 \%$ in studies in which this was reported as an outcome. Conclusions: Sulcus-deepening trochleoplasty performed for recurrent patellar instability in the setting of trochlear dysplasia results in improved Kujala scores and a low redislocation rate, when performed as an isolated procedure or in combination with other stabilization procedures. Greater-level evidence is needed to better evaluate the overall efficacy of this procedure in addressing patellar instability. Level of Evidence: Level of Evidence, IV; Systematic review of level III and IV studies
\end{abstract}

$\mathbf{P}$ atellar instability, a debilitating condition that affects approximately 6 of 100,000 individuals in the United States, has several anatomic etiologies, including medial patellofemoral ligament (MPFL) complex incompetence, trochlear dysplasia, an excessively externally rotated or proximally located tibial tuberosity, insufficiency of the vastus medialis obliqus and quadriceps musculature, and excessive internal rotation of the femur. ${ }^{1}$ Diverse procedures exist to address these

From the Department of Orthopaedic Surgery, University of California San Francisco, San Francisco, California, U.S.A.

The authors report the following potential conflicts of interest or sources of funding: N.K.P reports consultant for Orthopediatrics. B.T.F. reports Editorial Board: Journal of Shoulder Elbow Surgery and Current Reviews in Musculoskeletal Medicine and stockloptions in Kaliber. D.A.L. reports grants from the Arthroscopy Association of North America and American Orthopedic Society of Sports Medicine and research/education support from Arthrex, Smith $\theta$ Nephew; and Wright Medical/Tornier. Full ICMJE author disclosure forms are available for this article online, as supplementary material. differing etiologies, including repair or reconstruction of the MPFL, trochleoplasty, tibial tubercle osteotomy, and femoral osteotomy. The successful treatment of patellar instability continues to be a challenge, given the multiple etiologies that may be responsible for the pathology. Although MPFL reconstruction and tibial tubercle osteotomies are common approaches for patellar stabilization, trochlear dysplasia continues to be a risk factor for poor surgical outcomes in many series. ${ }^{2-6}$

Received December 27, 2019; accepted June 21, 2020

Address correspondence to Drew Anderson Lansdown, M.D., San Francisco Orthopaedic Institute, University of California, 1500 Owens St., San Francisco,CA 94158.E-mail: Drew.Lansdown@ucsf.edu

(C) 2020 THE AUTHORS. Published by Elsevier Inc. on behalf of the Arthroscopy Association of North America. This is an open access article under the CC BY-NC-ND license (http://creativecommons.org/licenses/by-nc-nd/4.0/). 2666-061X/191576

https://doi.org/10.1016/j.asmr.2020.06.009 
Trochlear dysplasia is defined functionally as shallowness of the trochlea that may predispose to patellar maltracking and instability with knee flexion. ${ }^{5,6}$ It has been reported to be present in more than $80 \%$ of patients with patellar instability. ${ }^{5}$ The following 4 anatomic variations based on radiographic evaluation of a lateral view of the knee have been described: presence of a crossing sign, which is present when the contour of the trochlear floor intersects with or protrudes anterior to the contour of the lateral femoral condyle (type A); a crossing sign with a supratrochlear spur (type B); a crossing sign with a double-contour sign reflecting a hypoplastic medial femoral condyle (type C); and absence of the trochlea, when all three signs are present (type D). ${ }^{5-7}$ In addition, on the Merchant view the sulcus angle can be calculated: an angle greater than $145^{\circ}$ is defined as dysplastic. ${ }^{6,8}$ Despite the high prevalence of trochlear dysplasia in patients with patellar instability, the surgical treatment of trochlear dysplasia with a trochleoplasty has been rarely used due to the highly technical nature of the procedure and some concern for disruption of the articular cartilage. ${ }^{9}$

There are 3 principal types of trochleoplasty: lateral facet elevation, sulcus deepening, and recession wedge, which are often performed in combination with bony or soft-tissue corrective procedures. ${ }^{2-4,6,9-12}$ The modern sulcus-deepening technique was described by Dejour in 1987 and additionally modified by Bereiter and Gautier in 1994. ${ }^{2,13-16}$ Dejour et al. suggested that the sulcus-deepening procedure may be most appropriate for type $\mathrm{B}$ and type D dysplasia, whereas type C dysplasia may be more amenable to the lateral facet-elevating technique. ${ }^{2,5,7}$ In the modified sulcusdeepening procedure described by Bereiter and Gautier, a cartilage "flake" is elevated in the trochlear groove and a burr or straight osteotome is used to remove the underlying bone to effectively deepen the trochlear groove, after which the cartilage flake is replaced and secured such that it plastically conforms over the con-

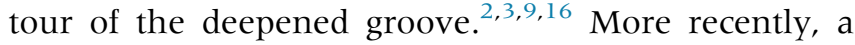
variation of this approach has been described in which a thick osteochondral flap is elevated rather than a thin cartilage flake. ${ }^{14}$

A sulcus-deepening technique is the most common form of trochleoplasty performed. ${ }^{9}$ Compared with other patellar-stabilization techniques such as MPFL reconstruction and tibial tubercle transfer, less evidence is available regarding trochleoplasty. The purpose of this systematic review is to highlight the indications and outcomes for sulcus-deepening trochleoplasty, when used as an isolated procedure as well as in combination with other stabilization techniques for patellar instability. Given the high prevalence of trochlear dysplasia among individuals with recurrent patellar instability, we hypothesize that trochleoplasty alone or in combination with additional bony or soft-tissue techniques will prove an effective method for reducing the occurrence of patellar instability and improving patient-reported outcomes.

\section{Methods}

A systematic review and meta-analysis were performed according to PRISMA (Preferred Reporting Items for Systematic Reviews and Meta-Analyses) guidelines.

\section{Study Eligibility}

Inclusion criteria were studies in the English language (levels of evidence 1-4) that evaluated standardized patient-reported outcome measures as well as recurrent dislocation or instability following trochleoplasty as a surgical treatment for patellar instability and/or trochlear dysplasia. Surgical treatment was set to include sulcus-deepening trochleoplasty as either an isolated or combined procedure used in treatment. Exclusion criteria included reviews of the literature, expert opinions, nonclinical studies, isolated case reports, and clinical series that did not involve commonly used, validated outcomes scoring systems or report on clinical and/or functional patient outcome measures.

\section{Literature Search}

An electronic search was performed in MEDLINE via PubMed and Embase. The search included the key words "trochleoplasty" OR "trochlear dysplasia." The final search was performed on March 20, 2020.

\section{Study Selection and Data Abstraction}

Three authors (M.R.D., T.E.D., R.D.F.) independently selected relevant articles based on title from the search results. The abstracts of all titles chosen by any one of the authors were then analyzed and data were recorded in spreadsheet format.

\section{Risk of Bias Assessment}

Study bias was analyzed using the Methodological Index for Non-Randomized Studies (MINORS) criteria, a validated instrument of assessment of nonrandomized studies, by 2 independent reviewers (M.R.D., S.A.). ${ }^{17}$

\section{Data Analysis}

Data extraction followed a standardized protocol developed before the search. The pertinent characteristics of each study were collected, including study design, year of publication, patient number, and level of evidence, as well as the characteristics of study participants (age, sex, primary vs revision surgery). Treatment technique was recorded, specifying the type of sulcusdeepening trochleoplasty performed, and primary clinical outcome measures including Kujala score and re-dislocation rate. Markers of clinical (physical examination findings and return-to-sport) and radiologic 
Table 1. Studies Meeting Inclusion Criteria

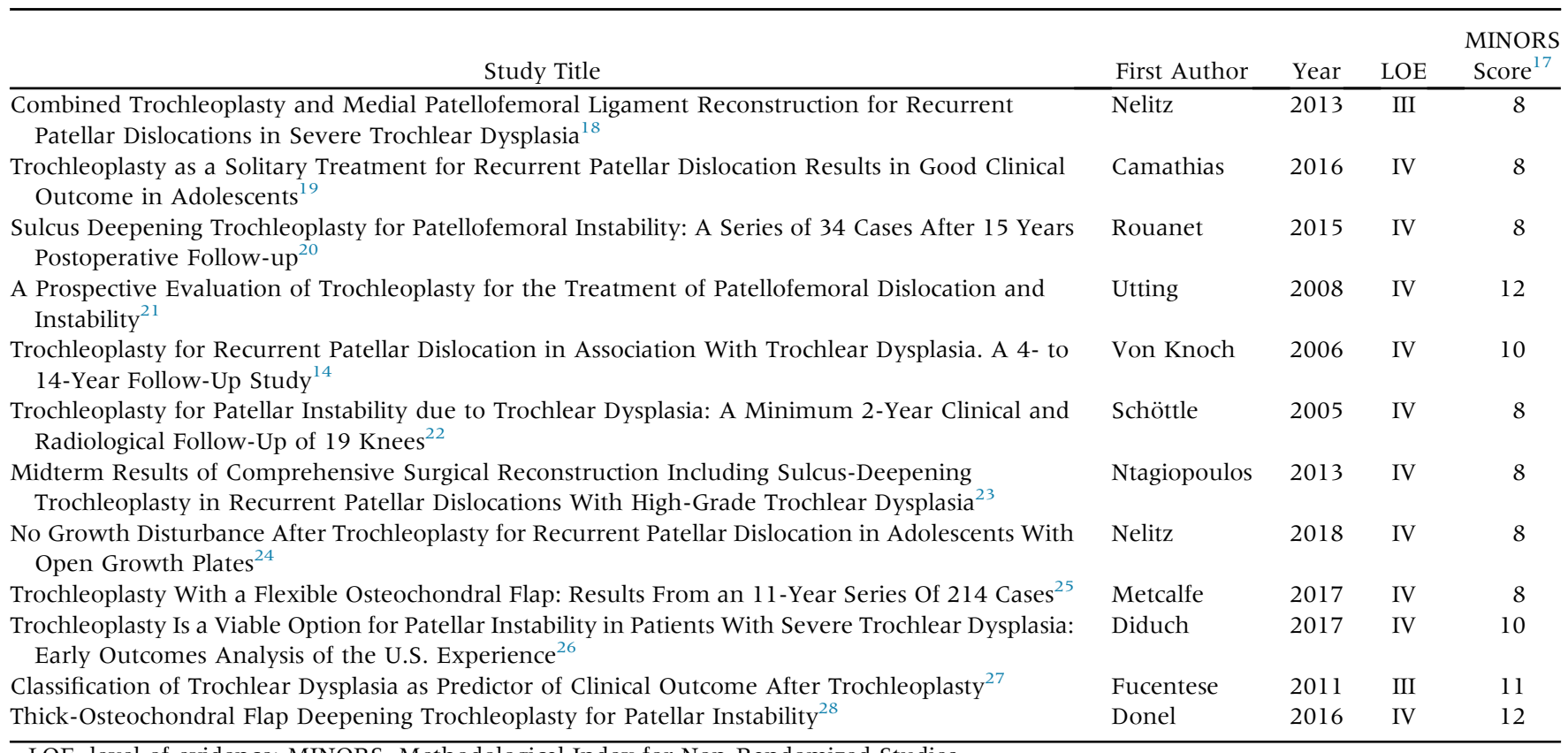

LOE, level of evidence; MINORS, Methodological Index for Non-Randomized Studies.

outcomes were also documented, in addition to complications. Given the heterogeneity of outcomes and low level of available evidence on this subject (Table 1), a formal meta-analysis could not be performed, although results of isolated trochleoplasty compared with trochleoplasty as part of a combined stabilization procedure were considered qualitatively. Redislocation rate following surgery was recorded, and the Kujala score was the most frequently reported clinical outcome measure.

\section{Results}

Using the study-acquisition algorithm detailed in Figure 1, we identified 888 studies related to the keywords "trochleoplasty" OR "trochlear dysplasia" and narrowed our search to 12 studies related to sulcusdeepening trochleoplasty and its impact on patientreported outcomes and patellar instability, as well as complications encountered ${ }^{14,18-28}$ (Table 1). Final studies were selected based on inclusion of all or most of the following metrics: (1) use of standardized patient-reported outcome scores such as the Kujala score, (2) inclusion of dislocation rates as an outcome measure, (3) inclusion of clinical outcome measures such as presence of a $\mathrm{J}$-sign or positive apprehension test, and (4) inclusion of any relevant complications related to the procedure. All studies had lower quality assessment as per MINORS criteria, with each study being non-comparative with a score $<16$ (Table 1). ${ }^{17}$ All included studies were either Level III or Level IV evidence (Table 1). Among the 12 studies included, there were 702 cases of trochleoplasty performed on
639 patients, $67 \%$ of whom were female (Table 2). Trochleoplasty was performed as a first-time surgical intervention in $74 \%$ of cases and was an isolated stabilization intervention in $33.9 \%$ of cases.

As the Kujala scoring questionnaire was the most widely used patient-reported clinical outcomes measure across studies, it was considered qualitatively between trochleoplasty that was performed as an isolated intervention and trocheoplasty as part of a combined stabilization procedure. Mean Kujala scores ranged from 81 to 92 postoperatively in the isolated trochleoplasty group and 76 to 95 in the combined group. Recurrent

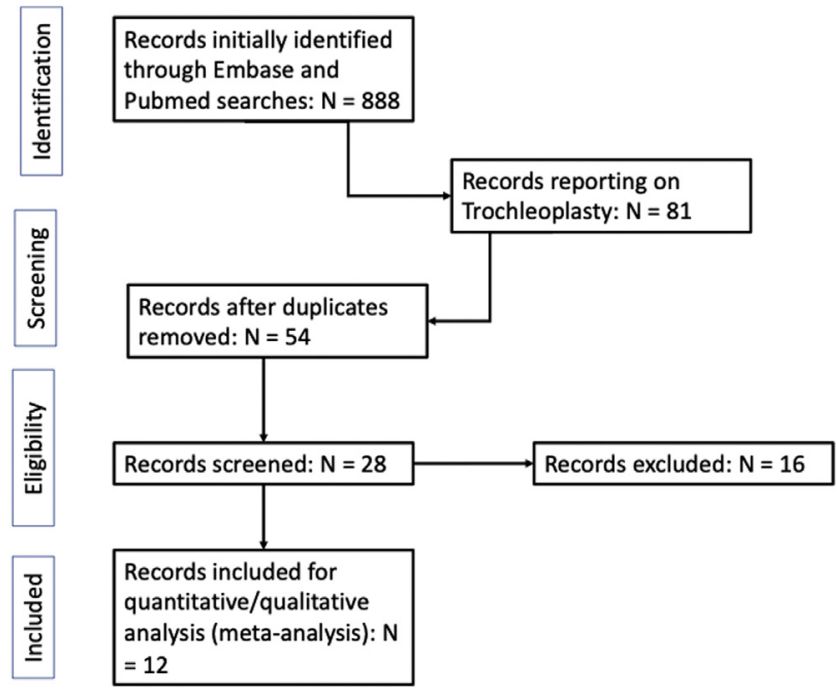

Fig 1. Study acquisition flowchart demonstrating the algorithm to reach the 12 studies ultimately included. 
Table 2. Demographic Baseline Data Including Sex, Preoperative Dislocations, and Type (Primary Versus Revision) of Procedure

\begin{tabular}{|c|c|c|c|c|}
\hline Study & $\begin{array}{l}\text { Patients } \\
\text { (Knees) }\end{array}$ & Sex & Dislocations Before Surgery & $\begin{array}{c}\text { Primary vs Revision } \\
\text { Stabilization Procedure }\end{array}$ \\
\hline Nelitz et al., $2013^{18}$ & $23(26)$ & $\begin{array}{l}\text { M: } 10 \\
\text { F: } 16\end{array}$ & $>2$ & $26 / 26$ primary \\
\hline Camathias et al., $2016^{19}$ & $44(50)$ & $\begin{array}{r}M: 20 \\
F: 30\end{array}$ & $\begin{array}{l}\text { Recurrent dislocations not responding to } \\
\text { nonoperative treatment for } 6 \text { mo }\end{array}$ & 50/50 primary \\
\hline Rouanet et al., $2015^{20}$ & 34 & $\begin{array}{l}\text { M: } 10 \\
\text { F: } 24\end{array}$ & $\begin{array}{l}\text { Average of } 6 \text { dislocations per patient } \\
\text { preoperatively }\end{array}$ & 21/34 primary; 13/34 revision \\
\hline Utting et al., $2008^{21}$ & $54(59)$ & $\begin{array}{l}\text { M: } 15 \\
\text { F: } 44\end{array}$ & Unspecified & $43 / 59$ primary, $16 / 59$ revision \\
\hline Von Knoch et al., $2006^{14}$ & $38(45)$ & $\begin{array}{l}\text { M: } 22 \\
\text { F: } 16\end{array}$ & Unspecified & $30 / 45$ primary, $15 / 45$ revision \\
\hline Schöttle et al., $2005^{22}$ & 19 & $\begin{aligned} M & : 4 \\
F & : 15\end{aligned}$ & $\begin{array}{l}\geq 2 \text { dislocations, or } 1+\text { persistent } \\
\text { apprehension sign }\end{array}$ & 14/19 primary, 5/19 revision \\
\hline Ntagiopoulos et al., $2013^{23}$ & $27(31)$ & $\begin{array}{l}\text { M: } 14 \\
\text { F: } 13\end{array}$ & $>3$ dislocations & Not stated \\
\hline Nelitz et al., $2018^{24}$ & $18(18)$ & $\begin{array}{l}\text { M: } 6 \\
\text { F: } 12\end{array}$ & $>2$ & 12/12 primary \\
\hline Metcalfe et al., $2017^{25}$ & $185(214)$ & $\begin{aligned} \text { M: } & 52 \\
\text { F: } & 133\end{aligned}$ & Unspecified & $154 / 214$ primary, 60 revision \\
\hline Diduch et al., $2017^{26}$ & $43(49)$ & $\begin{array}{r}\text { M: } 10 \\
\text { F: } 39\end{array}$ & Unspecified & 26/49 primary, 23/49 revision \\
\hline Fucentese et al., $2011^{27}$ & $38(44)$ & $\begin{array}{l}\text { M: } 11 \\
\text { F: } 33\end{array}$ & $\begin{array}{l}>2 \text { dislocations, or } 1 \text { with persistent } \\
\text { apprehension }\end{array}$ & $31 / 44$ primary, $13 / 44$ revision \\
\hline Donel et al., $2016^{28}$ & $90(107)$ & $\begin{array}{l}\text { M: } 36 \\
\text { F: } 54\end{array}$ & $\begin{array}{c}>1 \text { dislocation, failed conservative } \\
\text { management or prior surgery }\end{array}$ & 64/107 primary surgery, 43/107 revision \\
\hline
\end{tabular}

F, female; M, male.

dislocation was an overall rare occurrence, ranging from 0 to $8 \%$. No recurrent dislocations were reported in 7 of the studies reviewed $(\mathrm{N}=218$ total knees). ${ }^{14,18,20,22-24,26}$ Three studies reported a single recurrent dislocation. ${ }^{19,21,27}$ Metcalfe et al. ${ }^{25}$ reported a total of 16 recurrent dislocation events of 199 cases $(8 \%)$ in the largest case series included (Table 3).

Among the most common clinical metrics for persistent instability following trochleoplasty were presence of a J-sign or a positive apprehension test. Following surgery, 0 to $12 \%$ of patients had a persistent J-sign, and 0 to $29 \%$ exhibited a positive apprehension test (Table 4). Among studies that reported return to sport metrics, there was a return to play rate that ranged from $65 \%$ to $83 \% .^{18,21,25,26}$ Among the most frequently documented radiographic metrics was the progression of radiographic arthritis following trochleoplasty (Table 4). The presence of at least mild radiographic arthritis was documented in up to $97 \%$ of patients over 15 years after surgery by Rouanet et al., ${ }^{20}$ whereas Metcalfe et al. ${ }^{25}$ documented approximately $3 \%$ of cases that showed radiographic progression to arthritis. Von Knoch et al. ${ }^{14}$ and Schöttle et al. ${ }^{22}$ additionally assessed for the presence of a "crossing sign" as a radiographic indication of persistent trochlear dysplasia following surgery and found it to be present in 2 of 45 patients and 3 of 19 patients, respectively.

Reported complications were relatively infrequent across studies, with the most prevalent being persistent knee stiffness or loss of flexion after surgery (Table 5). Diduch et al. $^{26}$ reported an arthrofibrosis rate of $18 \%$ requiring manipulation under anesthesia in their series of 49 cases. Rouanet et al. $^{20}$ reported stiffness limiting flexion to less than $90^{\circ}$ in 8 of 34 patients $(24 \%)$. In addition, over the 15-year follow-up period, 6 of 34 knees were revised to either a patellofemoral or total knee arthroplasty, and 1 of 34 underwent revision by tibial tubercle osteotomy given persistent instability. ${ }^{20}$ Nelitz et al. ${ }^{24}$ in 2018 reported that 4 of 18 patients had either reduced flexion requiring further rehabilitation, adhesions requiring repeated arthroscopy, or a persistent flexion contracture of up to $5^{\circ}$ at final follow-up. Woundrelated complications were rare, reported in 2 of 59 cases by Utting et al. ${ }^{21}$ and 1 of 44 cases in Fucentese et al. ${ }^{27}$

\section{Discussion}

Overall, Kujala scores increased postoperatively compared with preoperatively in cases in which trochleoplasty was performed as an isolated or combined procedure. A 2019 case series of 211 isolated MPFL reconstructions reported an average Kujala score of 88.8 postoperatively. ${ }^{29}$ Similarly, a 2018 case series by Liu et al. ${ }^{30}$ of anteromedialization tibial tubercle osteotomy in 48 patients found average postoperative Kujala scores of 82.6. Thus, it appears that on average, Kujala scores for either isolated trochleoplasty or combined stabilization surgery involving trochleoplasty are similar to previously reported scores for other 
Table 3. Primary Outcomes Including Type of Procedure (Isolated Trochleoplasty Versus Combined Procedure), Kujala Scores, and Redislocation Rates

\begin{tabular}{|c|c|c|c|c|c|c|c|}
\hline Study & $\begin{array}{c}\text { No. Isolated } \\
\text { Trochleoplasty }\end{array}$ & $\begin{array}{c}\text { No. Combined } \\
\text { Procedures }\end{array}$ & $\begin{array}{l}\text { Kujala } \\
\text { (Isolated) }\end{array}$ & $\begin{array}{c}\text { Kujala } \\
\text { (Combined) }\end{array}$ & $\begin{array}{c}\text { Redislocation } \\
\text { (Isolated) }\end{array}$ & $\begin{array}{c}\text { Redislocation } \\
\text { (Combined) }\end{array}$ & $\begin{array}{c}\text { Mean } \\
\text { Follow-up } \\
\text { Length, y }\end{array}$ \\
\hline Nelitz et al., $2013^{18}$ & $0 / 26$ & $26 / 26$ & $\overline{-}$ & $\begin{array}{l}79 \text { preoperative } \\
90 \text { follow-up } \\
\text { Diff }=11\end{array}$ & - & $0 / 26(0 \%)$ & 2.5 \\
\hline Camathias et al., $2016^{19}$ & $50 / 50$ & $0 / 50$ & $\begin{array}{l}71 \text { preoperative } \\
92 \text { postoperative } \\
\text { Diff }=21\end{array}$ & - & $1 / 50(2 \%)$ & - & 2.75 \\
\hline Utting et al., $2008^{21}$ & $32 / 59$ & $27 / 59$ & - & $\begin{array}{l}62 \text { preoperative } \\
76 \text { postoperative } \\
\text { Diff }=14\end{array}$ & - & $1 / 59(1.7 \%)$ & 2 \\
\hline $\begin{array}{l}\text { Von Knoch et al., } \\
2006^{14}\end{array}$ & $0 / 45$ & $45 / 45$ & - & 94.9 & - & $0 / 45(0 \%)$ & 8.3 \\
\hline Schöttle et al., $2005^{22}$ & $19 / 19$ & $0 / 19$ & $\begin{array}{l}56 \text { preoperative } \\
80 \text { postoperative } \\
\text { Diff }=24\end{array}$ & - & $0 / 19(0 \%)$ & - & 3 \\
\hline Nelitz et al., $2018^{24}$ & $0 / 18$ & $18 / 18$ & - & $\begin{array}{l}67 \text { preoperative } \\
89.5 \text { postoperative } \\
\text { Diff }=22.5\end{array}$ & - & $0 / 18(0 \%)$ & 2.3 \\
\hline Metcalfe et al., $2017^{25}$ & $36 / 224$ & $188 / 224$ & - & $\begin{array}{l}51.5 \text { preoperative } \\
82.5 \text { postoperative } \\
\text { Diff }=31\end{array}$ & - & $16 / 199(8.0 \%)$ & 4.43 \\
\hline Diduch et al., $2017^{26}$ & $0 / 49$ & $49 / 49$ & - & $\begin{array}{l}54.5 \text { preoperative } \\
82.5 \text { postoperative } \\
\text { Diff }=28\end{array}$ & - & $0 / 49(0 \%)$ & 0.88 \\
\hline Fucentese et al., $2011^{27}$ & $44 / 44$ & $0 / 44$ & $\begin{array}{l}68 \text { preoperative } \\
90 \text { postoperative } \\
\text { Diff }=22\end{array}$ & - & $1 / 44(2.2 \%)$ & - & 4 (median) \\
\hline
\end{tabular}

Diff, difference; pre, preoperative; post, postoperative.

well-established stabilization techniques. Among the studies included in this review, more than one half of cases involved an isolated sulcus-deepening technique without other concurrent stabilization procedures, and surgery was more frequently performed as a primary surgical intervention than a revision surgery.

The shared purpose of trochleoplasty, MPFL reconstruction, and tibial tubercle osteotomy is to prevent redislocation of the patella and treat persistent instability. Thus, one important focus of this review was to assess redislocation rates, in addition to persistence of clinical instability as evidenced by the J-sign and positive apprehension test. We found that, overall, there was a low dislocation rate among all cases involving trochleoplasty as either an isolated or combined procedure, ranging from 0 to $8 \%$. In addition, we found that the persistence of a J-sign after surgery was a relatively rare occurrence, ranging from 0 to $12 \%$, whereas a positive patellofemoral apprehension test was more common postoperatively, ranging from 0 to
$29 \%$. One perceived benefit of trochleoplasty compared with other stabilization techniques is that it directly addresses the bony deformity involved in trochlear dysplasia that contributes to instability. However, a recent case series by Liu et al. ${ }^{31}$ of 121 isolated MPFL reconstructions performed in patients with Dejour B, C, or D trochlear dysplasia demonstrated mean Kujala scores of 90 and a total of 3 of 121 redislocations over an average follow-up of 44 months. Thus, even in patients with trochlear dysplasia, soft-tissue procedures may prove to be effective in improving clinical outcomes and preventing redislocation with appropriate patient selection.

A topic of recent interest is the use of trochleoplasty as an isolated versus combined procedure in addressing patellar instability. Ren et al. ${ }^{32}$ performed a systematic review of 192 cases comparing isolated trochleoplasty to that performed in conjunction with MPFL reconstruction, and found a significantly lower redislocation rate with the combined procedure. 
Table 4. Clinical (Including Physical Examination and Return to Sport) and Radiologic Outcomes

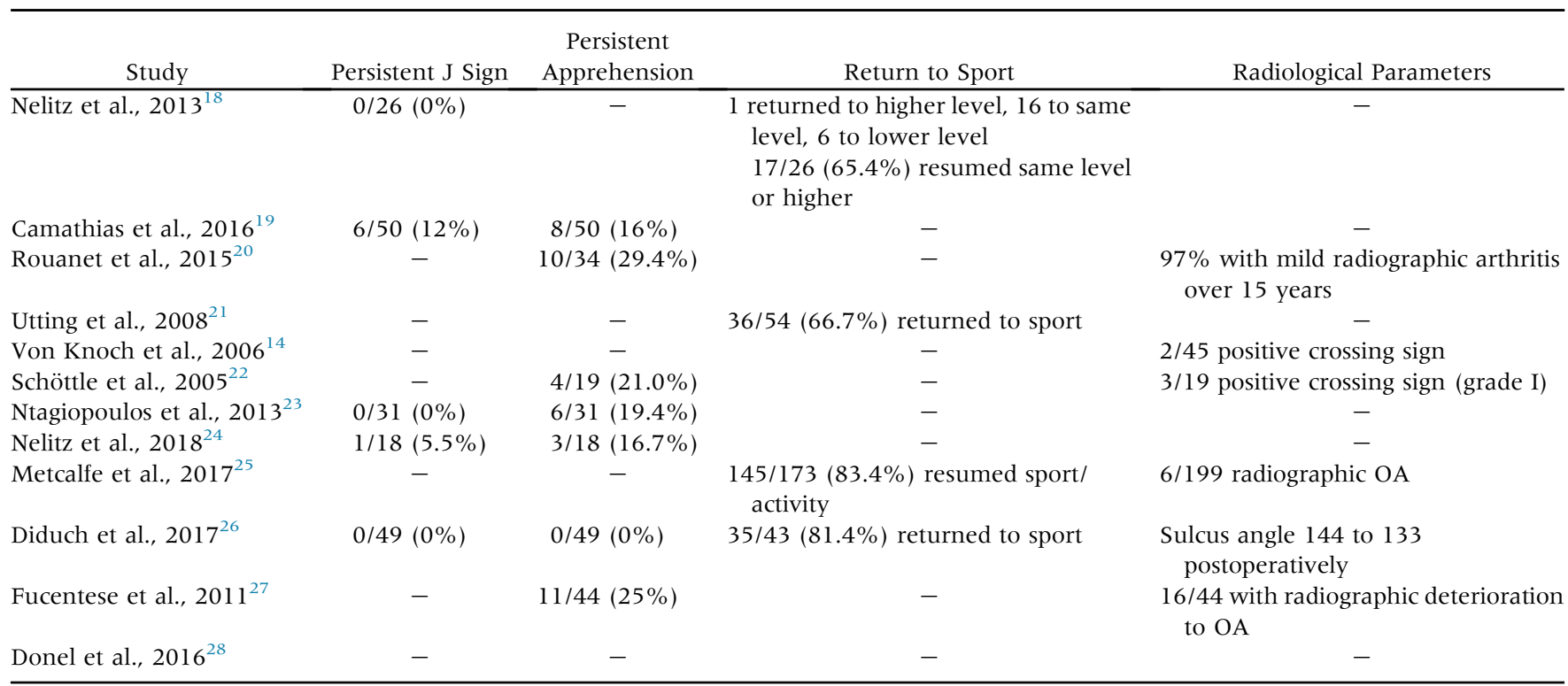

OA, osteoarthritis.

Balcarek et al. $^{33}$ further performed a meta-analysis comparing isolated MPFL reconstruction to MPFL reconstruction performed with trochleoplasty and similarly found that the combined procedure dislocation rate was significantly lower at $2.1 \%$ compared with $7 \%$ in the isolated MPFL group. The study by Metcalfe et al. ${ }^{25}$ included in this review is the largest case series of sulcus-deepening trochleoplasty performed to date, of which the majority of surgeries were performed as combined procedures involving trochleoplasty and a soft-tissue balancing procedure. Of note, this study also reported the highest redislocation rate postoperatively, at $8 \% .^{25}$ Nine of 16 of the redislocations occurred in the first half of the surgeries performed, and the remainder in the second half, suggestive that the effect of a technical learning curve, if present, is minimal according to the authors. ${ }^{25}$

Among the studies included, the most common indication for including trochleoplasty in a surgical intervention was recurrent patellar instability, frequently defined as greater than 2 dislocation events, or a single dislocation event with a persistent apprehension sign on examination. Additionally, most studies considered the radiographic presence of Dejour trochlear dysplasia in the setting of recurrent dislocations as an indication for trochleoplasty. A consideration against performing trochleoplasty as an isolated stabilization procedure is the risk of arthrofibrosis and decreased post-operative range of motion, as well as the risk of radiographic progression of patellofemoral arthritis that it carries. ${ }^{18-21,24,26,34}$ Although dislocation rates with trochleoplasty may be similar to other stabilization procedures such as MPFL reconstruction, the risk of limited range-of-motion and flexion contracture post-operatively may be more prevalent with trochleoplasty. Song et al. ${ }^{35}$ performed a systematic review of trochleoplasty compared with nontrochleoplasty procedures in 2014 and found that there was a lower patellar redislocation rate in procedures involving trochleoplasty as well as a lower percentage of radiographic patellofemoral osteoarthritis (Iwano grade 2 or greater) compared with nontrochleoplasty procedures; however, there was an inferior outcome with respect to range of motion at follow-up. In the present review, however, radiographic progression of arthritis was a commonly cited outcome after trochleoplasty, with Rouanet et al. ${ }^{20}$ noting $97 \%$ of patients having developed some degree of patellofemoral arthritis over the 15-year follow-up period, the longest follow-up period included in this review.

Given the risk of postoperative stiffness following trochleoplasty, postoperative rehabilitation protocols must balance protection of bony healing with adequate range of motion. While specific descriptions of rehabilitation protocols were not consistently included in the studies analyzed, Carstensen al. ${ }^{36}$ published a recent case series on postoperative arthrofibrosis following trochleoplasty, in which patients began physical therapy three days after their index procedure. During the first 2 weeks after surgery, patients were kept $50 \%$ weightbearing, after which time they were advanced to full weightbearing. Flexion was limited $0^{\circ}$ to $70^{\circ}$ for weeks 1 and 2 postoperatively, then advanced to $90^{\circ}$ of flexion for weeks 3 and 4, before being advanced to full range of motion. ${ }^{36}$ Even with the early initiation of this protocol, 11 of 62 knees developed arthrofibrosis and underwent manipulation under anesthesia within 3 months of the index procedure, with 9 of these patients subsequently requiring arthroscopic lysis of adhesion. Following 
Table 5. Reported Complications

\begin{tabular}{|c|c|}
\hline Study & Complications Reported \\
\hline Nelitz et al. $2013^{18}$ & $\begin{array}{l}\text { - Prolonged rehab }>6 \text { weeks to regain full } \\
\text { flexion: } 1 / 26 \text { knees }(3.8 \%)\end{array}$ \\
\hline $\begin{array}{l}\text { Camathias et al. } \\
2016^{19}\end{array}$ & $\begin{array}{l}\text { - Reoperation for arthroscopic lysis of } \\
\text { adhesions: } 4 / 50(8 \%) \\
\text { - Redislocation: } 1 / 50(2 \%)\end{array}$ \\
\hline $\begin{array}{l}\text { Rouanet et al. } \\
2015^{20}\end{array}$ & $\begin{array}{l}\text { - Postoperative stiffness: } 8 / 34(23.5 \%) \\
\text { - Manipulation under anesthesia: } 6 / 34(17.6 \%) \\
\text { - Revision to knee arthroplasty over } \\
\text { 15-year follow-up: } 6 / 34(17.6 \%) \\
\text { - Tibial tubercle osteotomy for persistent pain/ } \\
\text { instability: } 1 / 34(2.9 \%)\end{array}$ \\
\hline $\begin{array}{l}\text { Utting et al. } \\
2008^{21}\end{array}$ & $\begin{array}{l}\text { - Superficial wound infection: } 2 / 59(3.4 \%) \\
\text { - Redislocation: } 1 / 59(1.7 \%) \\
\text { - Manipulation under anesthesia: } 1 / 59(1.7 \%) \\
\text { - Anaphylactic reaction to intraoperative } \\
\text { antibiotic: } 1 / 59(1.7 \%)\end{array}$ \\
\hline $\begin{array}{l}\text { Von Knoch et al. } \\
2006^{14}\end{array}$ & $\begin{array}{l}\text { - Patella baja: } 1 / 45(2.2 \%) \\
\text { - Persistent subluxation requiring distal } \\
\text { realignment osteotomy: } 1 / 45(2.2 \%)\end{array}$ \\
\hline $\begin{array}{l}\text { Schöttle et al. } \\
2005^{22}\end{array}$ & $\begin{array}{l}\text { - No complications explicitly reported for } \\
19 / 19 \text { knees }(0 \%)\end{array}$ \\
\hline $\begin{array}{l}\text { Ntagiopoulos et al. } \\
2013^{23}\end{array}$ & $\begin{array}{l}\text { - Staple breakage, removed arthroscopically: } \\
2 / 31(6.5 \%) \\
\text { - Deep venous thrombosis, treated with } \\
\text { anticoagulation: } 2 / 31(6.5 \%)\end{array}$ \\
\hline Nelitz et al. $2018^{24}$ & $\begin{array}{l}\text { - Flexion contracture up to } 5^{\circ}: 2 / 18(11.1 \%) \\
\text { - Reduced flexion treated with intense } \\
\text { rehabilitation: } 1 / 18(5.6 \%) \\
\text { - Reoperation for arthroscopic lysis of } \\
\text { adhesions: } 1 / 18(5.6 \%)\end{array}$ \\
\hline $\begin{array}{l}\text { Metcalfe et al. } \\
\qquad 2017^{25}\end{array}$ & $\begin{array}{l}\text { - } \text { Reoperation: } 27 / 224(12.1 \%) \\
\text { - Partial detachment of cartilage flap, treated } \\
\text { with debridement and microfracture at } \\
6 \text { weeks postoperatively: } 1 / 224(0.45 \%) \\
\text { - Complex regional pain syndrome: } \\
1 / 224(0.45 \%) \\
\text { - Foot drop despite normal } \mathrm{MRI} / \text { nerve } \\
\text { conduction studies: } 1 / 224(0.45 \%)\end{array}$ \\
\hline $\begin{array}{l}\text { Diduch et al. } \\
\qquad 2017^{26}\end{array}$ & $\begin{array}{l}\text { - Arthrofibrosis requiring manipulation under } \\
\text { anesthesia: } 9 / 49(18.4 \%)\end{array}$ \\
\hline $\begin{array}{l}\text { Fucentese et al. } \\
\qquad 2011^{27}\end{array}$ & $\begin{array}{l}\text { - } \text { Repeat arthroscopy for ongoing pain: } \\
3 / 44(6.8 \%) \\
\text { - } \text { Residual instability requiring further } \\
\text { stabilization procedures: } 2 / 44(4.5 \%) \\
\text { - New atraumatic dislocation postoperatively: } \\
1 / 44(2.3 \%) \\
\text { - Loose body removal: } 1 / 44(2.3 \%) \\
\text { - Transient postoperative femoral nerve palsy: } \\
\text { - } 1 / 44(2.3 \%) \\
\text { - } \text { Pound-healing complication: } 1 / 44(2.3 \%) \\
\text { syndrome: } 1 / 44(2.3 \%)\end{array}$ \\
\hline Donel et al. $2016^{28}$ & - No complications reported $(0 \%)$ \\
\hline
\end{tabular}

MRI, magnetic resonance imaging.

these interventions, there was no significant difference in range of motion between the arthrofibrotic and non-arthrofibrotic knees at final mean follow-up of 32.5 months. $^{36}$

Patellofemoral instability is multifaceted in its underlying pathogenesis compared with many orthopaedic conditions, and thus it deserves an individualized treatment approach that carefully takes into account the pathoanatomy and biomechanics that result in an individual's recurrent patellar dislocations. Based on the present data, we would recommend cautious consideration of trochleoplasty as part of an individualized, combination approach to patellar stabilization in patients with refractory instability and evident trochlear dysplasia, for whom an isolated soft-tissue procedure such as MPFL reconstruction may not fully address their underlying pathoanatomy resulting in instability.

\section{Limitations}

This review has several limitations. Trochleoplasty as a treatment for patellofemoral instability is relatively sparse compared with other more common stabilization techniques such as MPFL reconstruction and tibial tubercle osteotomy, and thus the studies included were all nonrandomized case series. Due to the relative lack of higher-level studies currently available and inconsistent data reporting, a meta-analysis could not be performed, and weighted averages of outcome measures could not be presented due to the risk of introducing bias. Given the nonrandomized nature of the cases included in the review, it is not possible to control for all variables that may contribute to the outcomes reported. There is also heterogeneity in the surgical technique in sulcusdeepening trochleoplasty between studies, with differences in the shape of the bony resection between the techniques described by Masse, Dejour et al., and Bereiter and Gautier, as well as variations in the use of a thin cartilage flake compared with a thick osteochondral flap. ${ }^{2,5,7,12,13,15,16}$ Studies frequently lacked detail regarding individual patient pathology that resulted in treatment with trochleoplasty with or without other stabilization procedures, so the specific indications for trochleoplasty remain difficult to elucidate given the available data. In addition, given the highly technical nature of trochleoplasty, which frequently necessitates a specialized center and expert, high-volume surgeon, the results reported by the studies included may not reflect those of the community-at-large. Overall, however, this review represents a large collection of consistently reported data that help give a broader view of the patient-reported and clinical outcomes and complications of sulcus-deepening trochleoplasty.

\section{Conclusions}

Sulcus-deepening trochleoplasty performed for recurrent patellar instability in the setting of trochlear dysplasia results in improved Kujala scores and a low redislocation rate, when performed as an isolated procedure or in combination with other stabilization procedures. Greater-level evidence is needed to better evaluate the overall efficacy of this procedure in addressing patellar instability. 


\section{References}

1. Fisher B, Nyland J, Brand E, Curtin B. Medial patellofemoral ligament reconstruction for recurrent patellar dislocation: A systematic review including rehabilitation and return-to-sports efficacy. Arthroscopy 2010;26: 1384-1394.

2. Dejour D, Saggin P. The sulcus deepening trochleoplastythe Lyon's procedure. Int Orthop 2010;34:311-316.

3. Nolan JE, Schottel PC, Endres NK. Trochleoplasty: Indications and technique. Curr Rev Musculoskelet Med 2018;11:231-240.

4. Albee F. The bone graft wedge in the treatment of habitual dislocation of the patella. Med Rec 1915;88: 257-259.

5. Dejour H, Walch G, Nove-Josserand L, Guier C. Factors of patellar instability: An anatomic radiographic study. Knee Surg Sports Traumatol Arthrosc 1994;2:19-26.

6. LaPrade RF, Cram TR, James EW, Rasmussen MT. Trochlear dysplasia and the role of trochleoplasty. Clin Sports Med 2014;33:531-545.

7. Dejour D, Le Coultre B. Osteotomies in patello-femoral instabilities. Sports Med Arthrosc Rev 2007;15:39-46.

8. Malghem J, Maldague B. Depth insufficiency of the proximal trochlear groove on lateral radiographs of the knee: Relation to patellar dislocation. Radiology 1989;170: 507-510.

9. Ntagiopoulos PG, Dejour D. Current concepts on trochleoplasty procedures for the surgical treatment of trochlear dysplasia. Knee Surg Sports Traumatol Arthrosc 2014;22: 2531-2539.

10. Blønd L, Schöttle PB. The arthroscopic deepening trochleoplasty. Knee Surg Sports Traumatol Arthrosc 2010;18: 480-485.

11. Goutallier D, Raou D, Van Driessche S. Retro-trochlear wedge reduction trochleoplasty for the treatment of painful patella syndrome with protruding trochleae. Technical note and early results. Rev Chir Orthop Reparatrice Appar Mot 2002;88:678-685 [in French].

12. Masse Y. Trochleoplasty. Restoration of the intercondylar groove in subluxations and dislocations of the patella. Rev Chir Orthop Reparatrice Appar Mot 1978;64:3-17 [in French].

13. Batailler C, Neyret P. Trochlear dysplasia: imaging and treatment options. EFORT Open Rev 2018;3:240-247.

14. von Knoch F, Böhm T, Bürgi ML, von Knoch M, Bereiter H. Trochleaplasty for recurrent patellar dislocation in association with trochlear dysplasia. A 4- to 14-year follow-up study. J Bone Joint Surg Br 2006;88: $1331-1335$.

15. Dejour H, Walch G. Pathologie fémoro-patellaire, Gème Journée Lyonnaise de Chirurgie du Genou. Lyon: Sauramps Medical, 1987.

16. Bereiter H, Gautier E. The trochleaplasty as a surgical therapy of recurrent dislocation of the patella in dysplastic trochlea of the femur. Arthroskopie 1994;7:281-286.

17. Slim K, Nini E, Forestier D, Kwiatkowski F, Panis Y, Chipponi J. Methodological index for non-randomized studies (minors): Development and validation of a new instrument. ANZ J Surg 2003;73:712-716.

18. Nelitz M, Dreyhaupt J, Lippacher S. Combined trochleoplasty and medial patellofemoral ligament reconstruction for recurrent patellar dislocations in severe trochlear dysplasia: A minimum 2-year follow-up study. Am J Sports Med 2013;41:1005-1012.

19. Camathias C, Studer K, Kiapour A, Rutz E, Vavken P. Trochleoplasty as a solitary treatment for recurrent patellar dislocation results in good clinical outcome in adolescents. Am J Sports Med 2016;44:2855-2863.

20. Rouanet T, Gougeon F, Fayard JM, Rémy F, Migaud H, Pasquier G. Sulcus deepening trochleoplasty for patellofemoral instability: A series of 34 cases after 15 years postoperative follow-up. Orthop Traumatol Surg Res 2015;101:443-447.

21. Utting MR, Mulford JS, Eldridge JDJ. A prospective evaluation of trochleoplasty for the treatment of patellofemoral dislocation and instability. J Bone Joint Surg $\mathrm{Br}$ 2008;90:180-185.

22. Schöttle PB, Fucentese SF, Pfirrmann C, Bereiter H, Romero J. Trochleaplasty for patellar instability due to trochlear dysplasia: A minimum 2-year clinical and radiological follow-up of 19 knees. Acta Orthop 2005;76: 693-698.

23. Ntagiopoulos PG, Byn P, Dejour D. Midterm results of comprehensive surgical reconstruction including sulcusdeepening trochleoplasty in recurrent patellar dislocations with high-grade trochlear dysplasia. Am J Sports Med 2013:41:998-1004.

24. Nelitz M, Dreyhaupt J, Williams SRM. No growth disturbance after trochleoplasty for recurrent patellar dislocation in adolescents with open growth plates. Am J Sports Med 2018;46:3209-3216.

25. Metcalfe AJ, Clark DA, Kemp MA, Eldridge JD. Trochleoplasty with a flexible osteochondral flap: Results from an 11-year series of 214 cases. Bone Joint J 2017;99-B: 344-350.

26. Diduch DR, Burrus MT, Cancienne JM, et al. Trochleoplasty is a viable option for patellar instability in patients with severe trochlear dysplasia: Early outcomes analysis of the U.S. experience. Orthop J Sports Med 2017;5: 232596711750038 (7_suppl6).

27. Fucentese SF, Zingg PO, Schmitt J, Pfirrmann CWA, Meyer DC, Koch PP. Classification of trochlear dysplasia as predictor of clinical outcome after trochleoplasty. Knee Surg Sports Traumatol Arthrosc 201 1;19:1655-1661.

28. Donel S, Ali K, Smith DrT, McNamara I. Thick-osteochondral flap deepening trochleoplasty for patellar instability. Orthop J Sports Med 2016;4:232596711650004 (3_suppl 2).

29. Sappey-Marinier E, Sonnery-Cottet B, O'Loughlin P, et al. Clinical outcomes and predictive factors for failure with isolated MPFL reconstruction for recurrent patellar instability: A series of 211 reconstructions with a minimum follow-up of 3 years. Am J Sports Med 2019;47: 1323-1330.

30. Liu JN, Wu H-H, Garcia GH, Kalbian IL, Strickland SM, Shubin Stein BE. Return to sports after tibial tubercle osteotomy for patellofemoral pain and osteoarthritis. Arthroscopy 2018;34:1022-1029.

31. Liu JN, Brady JM, Kalbian IL, et al. Clinical outcomes after isolated medial patellofemoral ligament reconstruction for patellar instability among patients with trochlear dysplasia. Am J Sports Med 2018;46:883-889. 
32. Ren B, Zhang X, Zhang L, et al. Isolated trochleoplasty for recurrent patellar dislocation has lower outcome and higher residual instability compared with combined MPFL and trochleoplasty: A systematic review. Arch Orthop Trauma Surg 2019;139:1617-1624.

33. Balcarek P, Rehn S, Howells NR, et al. Results of medial patellofemoral ligament reconstruction compared with trochleoplasty plus individual extensor apparatus balancing in patellar instability caused by severe trochlear dysplasia: Aa systematic review and meta-analysis. Knee Surg Sports Traumatol Arthrosc 2017;25:3869-3877.
34. Verdonk R, Jansegers E, Stuyts B. Trochleoplasty in dysplastic knee trochlea. Knee Surg Sports Traumatol Arthrosc 2005;13:529-533.

35. Song G-Y, Hong L, Zhang H, et al. Trochleoplasty versus nontrochleoplasty procedures in treating patellar instability caused by severe trochlear dysplasia. Arthroscopy 2014;30:523-532.

36. Carstensen SE, Feeley SM, Diduch DR. Manipulation under anesthesia with lysis of adhesions is effective in arthrofibrosis after sulcus-deepening trochleoplasty: A prospective study. Orthop J Sports Med 2019;7:2325967119864868. 